\title{
ANATOMIA COMPARATIVA DEL XILEMA DEL TRONCO Y DE LA RAMA DE Cedrelinga catenaeformis Ducke (FABACEAE)
}

Heiter Valderrama Freyre*

\section{RESUMEN}

Muestras de madera del tronco y discos de $5 \mathrm{~cm}$ de espesor de las ramas fueron coleccionados de árboles en pie de la especie cedrelinga catenaeformis Ducke, para realizar un estudio comparativo de la estructura anatómica del xilema entre ambas partes del árbol, lo cual permitirá encontrar características comunes y diferenciales entre el tronco y la rama, que servirán como elementos de identificación y clasificación de la especie, sin precisar derribar el árbol.

Existen características cualitativas y cuantitativas comunes en ambas partes del árbol, que pueden ser utilizadas como elementos de identificación y clasificación anatómica de la madera del tronco, basado en la rama de $10 \mathrm{~cm}$ de diámetro. La clasificación anatómica de la madera, por sus características cuantitativas, fue similar en ambas partes del árbol; así mismo, por el tamaño de fibras, la madera el tronco y de las ramas fue calificada como apta para fabricar pulpa para papel.

Palabras Claves: Anatomía de maderas, xilema del tronco, xilema de ramas, Cedrelinga catenaeformis Ducke.

\begin{abstract}
In order to find similar and differentiation characteristicsin stem and branch xylem, which can be used as identification and classification elements whithout cutting down the tree, flakes from stem and $5 \mathrm{~cm}$ width disks from branch of stands of forest.
\end{abstract}


Species Cedrelinga catenaeformis Ducke, were collected to carry out a comparative study of the anatomical structure of the xylem of the stem and branch of the tree.

As similar qualitative and quantitative characteristics were found in both stem and branch xylem, they can be used as anatomical identification and classification elements for wood from stem, on the basic of $10 \mathrm{~cm}$ branch diameter. The wood anatomical classification based on its quantitative characteristics was similar in both parts of the tree. According to the fiber size, wood from stem and branches of Cedrelinga Catenaeformis Ducke was determined as good in fabrication of pulp for paper.

Key words: Wood anatomy, stem xylem, branch xylem, Cedreling catenaeformis Ducke.

\section{INTRODUCCION}

Actualmente con la declaración de Río de Janeiro (ECO-92), así como también de otras reuniones internacionales sobre la preservación de la Amazonía y control de medio ambiente, las entidades financiadotas están dejando de lado proyectos que tiene carácter destructivo de la floresta. En ese sentido, los proyectos relacionados con la estructura celular del xilema del tronco, para desarrollar sus actividades precisan derribar árboles, lo que ocasiona complicaciones de nivel ecológico que difícilmente tienen posibilidades de financiamiento.

Las muestras de madera se obtienen de árboles derribados, constituyendo una limitación para el estudio, al generar una fuerte inversión económica, que depende en gran parte del nivel de muestreo, siendo un árbol por especie al nivel semidetallado y cinco a más al nivel detallado (Copant, 1973).

Las investigaciones en anatomía de maderas comprenden el estudio de las características organolépticas, macroscópicas. Paula (1980), Camacho \& Canessa (1981), Trujillo \& Conzales (1985), entre otros autores, consideran que de acuerdo con sus fines, la Anatomía de la madera se divide en : Anatomía descriptiva y Anatomía tecnológica. Valderrama (1992) menciona que la anatomía descriptiva estudia características importantes de la madera con fines de identificación de la especie. La anatomía tecnológica estudia la influencia de la estructura anatómica en las propiedades tecnológicas de la madera con el fin de 
solucionar problemas técnicos de secado, preservación, trabajo con máquinas de carpintería, fabricación de pulpa para papel y en muchas otras aplicaciones.

La variabilidad de los factores de crecimiento del árbol origina la variación del tamaño de los elementos del xilema en un mismo árbol a un mismo nivel y diferentes niveles del tronco y de las ramas, entre árboles de la misma especie y entre especies, afectando a la madera durante el secado, preservado, resistencia y fabricación de pulpa para papel, entre otros comportamientos.

Muchas maderas tropicales son utilizadas como fuente de materia prima para fabricar papel y en la industria de la construcción, pero también existen especies desconocidas por sus usos. Las dimensiones, proporción, frecuencia y distribución de las fibras, vasos del tronco y ramas del árbol tienen relación directa o indirecta en el peso específico y por consiguiente en las características de resistencia de la madera y en la calidad de pulpa para papel (Klungness \& Sanyer, 1981; Maeglin \& Quirk, 1984; Kort, 1990; Wright \& Malan, 1991; Valderrama, 1992). Sin embargo, a pesar de que las dimensiones de fibras del tronco (Bhat \& Dhamodaran, 1989), la variación de las fibras en el sentido transversal y longitudinal encontrado en el xilema del tronco del árbol, puede o no tener relación con la variabilidad de la estructura celular de la madera en las ramas, así como la asociación entre ambas partes del árbol, de la probabilidad de encontrar características anatómicas en las ramas que se relacionan con la estructura celular y el uso de la madera del tronco (Denne \& Dodd, 1989).

Los anatomistas en madera buscan nuevos métodos que empleen procedimientos menos destructivos de árboles para caracterizar especies forestales por sus cualidades de uso.

Por otro lado, la variación cuantitativa de la estructura anatómica encontrada en el xilema a diferentes niveles del tronco, puede o no tener relación con la variabilidad de la estructura celular de la madera de las ramas.

En la actualidad, los datos existentes sobre el xilema de las ramas son limitados, la mayoríade los estudios son con especies de zonas templadas, donde muestran diferencias de la madera en una misma rama (Park et al., 1979; 1980 Lee \& Eom, 1988). Así, Manwiller (1974) encontró que la longitud de las fibras en ramas fueron significativamente cortos, variando de 0,66 mm (Hacer rubrum L) a 1,40 $\mathrm{mm}$ (Niza sylvatica $\mathrm{M}$ ); el promedio de la longitud de fibras en las ramas fue 24,4\% menor que en el tronco. Iqbal \& Ghouse (1983) en árboles de Acacia nilotica (L) W. var. Telia y Prosopis spicigera L., encontraron que el tamaño de 
las fibras del floema también longitud de fibras fue menor en las ramas mas jóvenes (parte superior de la ramificación) en relación a la longitud de fibras de las ramas de mayor diámetro (cerca al fuste).

Bhat \& Dhamodaran (1989) estudiando 11 maderas de especies foliosas, verificaron que la longitud de fibras en las ramas fueron consistentemente cortas en relación a las fibras del tronco.

Stoke \& Manwiller (1994) determinaron la proporción de vasos, fibras, radios, parénquima axial en el tronco, rama y madera de la raíz de Quercus velutina Lam ., y encontraron elevada proporción de elementos de vasos y fibras en las ramas. Además, difrencias estadísticamente significativas en la proporción de elementos de la madera entre localizaciones del árbol (ramas, troncos, raíz).

También page (1993) analizó las características anatómicas del xilema secundario del tronco, y de la rama, donde la forma, tamaño, distribución de la madera primaveril y la frecuencia de poros solitarios fueron los mas significativos. Los anillos de crecimiento son angostos en las ramas.

Cedrelinga catenaeformis D. es una especie monotípica. Se trata de un genero restringido a la región neotropical y tiene a la Amazonía como su centro de distribución natural (Freitas et all., 1992, Aróstegui \& Díaz, 1992). Árbol grande, de copa estrecha, perfecta y dominante, cuyo aspecto de la corteza es similar al del cedro (Cedrela odorata). De gran tamaño entre los árboles amazónicos, puede alcanzar 50 metros de altura y dos metros de diámetro a la altura del pecho (spichiger et al., 1989; Linares, 1986; Freitas et al., 1992). Los estudios silviculturales recomiendan usarlo en plantaciones en la Amazonía por presentar buen crecimiento en altura, diámetro, alto porcentaje de sobrevivencia en sol y sobre sombra, la especie puede ser utilizada para reforestar áreas abandonadas después de cultivos sucesivos de ciclo rápido (Alentar \& Araujo, 1971; Magalhaes \& Blum, 1984; Freitas et al., 1992).

El presente trabajo pretende contribuir con informaciones sobre la estructura celular de la madera del tronco y de la rama con diez centímetros de diámetro, de árboles de Cedrelinga catenaeformis Ducke (Fabaceae), como primer paso para establecer correlacionar al nivel anatómico entre el xilema del tronco y de las ramas del árbol, procurando encontrar características comunes que puedan servir como elementos de identificación y caracterización tecnológica de especies forestales a partir de esa parte del árbol. Para el desarrollo de esta investigación se consideró los siguientes objetivos: 


\section{GENERAL}

Realizar el estudio de la estructura celular del tejido xilemático del tronco y de la rama de Cedrelinga catenaeformis Ducke.

\section{ESPECIFICOS:}

- Determinar las características anatómicas cualitativas y cuantitativas comunes y diferenciales del tronco y de las ramas.

- Realizar la calificación tecnológica comparativa de la madera del tronco y de la rama para fabricación de pulpa para papel, basado en las dimensiones de fibras.

\section{MATERIAL Y METODOS}

La colecta y muestreo de madera fueron realizados de árboles seleccionados del Arboreto del Centro de Investigación y Enseñanza Forestal Puerto Almendras, de la Universidad Nacional de la Amazonía Peruana, Iquitos-Perú. El área está localizada en las coordenadas geográficas $04^{\circ} 05^{\prime}$ latitud sur y $73^{\circ} 40^{\prime}$ longitud oeste a una altitud media de 120 metros sobre el nivel del mar (ONERN, 1975). Políticamente se encuentra en la Amazonía Peruana, Provincia de Maynas, Región de Loreto y es parte de la formación vegetal del Bosque Húmedo Tropical (Tosi, 1960; ONERN, 1976). La precipitación anual es: media $2480 \mathrm{~mm}$., máxima $3471 \mathrm{~mm}$., mínima $1333 \mathrm{~mm}$.; temperatura mensual: media $26^{\circ} \mathrm{C}$, máxima $34^{\circ} \mathrm{C}$, mínima $17,5^{\circ} \mathrm{C}$; características que hacen que el área tenga clima húmedo y caliente, siendo muy húmedo en los meses de enero a mayo (ONERN, 1975).

La fase de laboratorio (preparación de muestras, montaje de fibras y tejidos, descripción, medición de células, microfotografías) fue realizada en las instalaciones del laboratorio de anatomía de maderas Facultad de Ingeniería Forestal- UNAP (Iquitos, Perú), con apoyo de la división de Anatomía de la Madera del Centro de Investigación de la Amazonía (INPA- Manaos).

Fueron seleccionados en el área de estudio cinco (5) árboles representativos de Cedrelinga catenaeformis Ducke ( Copant, 1972). De cada árbol en pie, al diámetro la altura del pecho (Dap), fueron cortadas muestras de madera de aproximadamente $10 \mathrm{~cm}$ de espesor; conteniendo parte de albura y duramen, de igual modo, fueron elegidas al azar dos (2) ramas de $10 \mathrm{~cm}$ de diámetro por árbol. El número de muestras fue: 05 del tronco y 20 de las ramas. 
Para la descripción de las características organolépticas de la madera fueron utilizadas probetas de xiloteca y cubos de madera en una pulgada de arista, elaboradas en las muestras colectadas. Para el estudio de las características microscópicas fueron utilizados cortes histológicos de las secciones transversal, tangencial y radial, obtenidos mediante procedimientos microtécnicos de maderas.

La meditación de elementos fibrosos y vasculares de cada muestra fue realizada en láminas preparadas con células aisladas. Las dimensiones de fibras (longitud, diámetro total, diámetro de lumen, espesor de pared celular), longitud diámetro tangencial frecuencia de los elementos vasculares y dimensiones de los radios fueron obtenidas utilizando un microscopio de luz a diferentes aumentos del ocular y del objetivo (4x, 10x, 40x), las medias fueron determinadas con escala del ocular y de la platina micrométrica, con una precisión de $1 / 1000 \mathrm{~mm}$. El número de mediciones fue de 25 por cada muestra. El análisis estadístico, fue realizado basado en las determinaciones de los valores promedios, desviación estándar y coeficiente de variación. También fueron realizados comparaciones de medias, mediante pruebas de hipótesis con $5 \%$ de probabilidad estadística.

La madera por sus características anatómicas cualitativas y cuantitativas en el tronco y rama, fue descrita y clasificada de acuerdo con terminologías propuestas por la IAWA, (1964; 1989); Copant, (1973) y Valderrama et al., (1989).

La evaluación tecnológica para la fabricación de pulpa para papel, basada en las fibras de la madera de ambas partes del árbol, fue realizada mediante el factor Runkel $(\mathrm{R}=2 \mathrm{e} / \mathrm{d})$, que relaciona el espesor de la pared celular con el diámetro del lumen y clasifica a la madera de acuerdo con los siguientes rangos:

\begin{tabular}{|c|c|l|}
\hline Grupo & R & \multicolumn{1}{|c|}{ Clasificación } \\
\hline I & $0-0,25$ & Excelente para papel \\
II & $0,26-0,50$ & Muy buena para papel \\
III & $0,51-1,0$ & Buena para papel \\
IV & $1,1-2,0$ & Regular para papel \\
V & 2,0 a más & Mala para papel \\
& & \\
\hline
\end{tabular}

Fuente: Camacho \& Canessa (1981) 


\section{RESULTADO Y DISCUSIÓN}

El método utilizado para la descripción y clasificación de la madera ha servido para definir las características cualitativas y cuantitativas de ambas partes del árbol.

\section{Estructura anatómica el tronco}

\section{Características Generales:}

Madera con densidad básica media $\left(0,43 \mathrm{gr} / \mathrm{cm}^{3}\right)$. En condición verde, albura de color pardo -claro, duramen pardo rosado. Seco al aire la coloración se mantiene. Olor y sabor no diferenciado. Brillo medio. Veteado en arcos superpuestos. Grano recto. Textura gruesa. Anillos de crecimiento diferenciados como bandas oscuras e irregulares.

\section{Características Macroscópicas:}

Poros visibles a simple vista, semicirculares a difusos, solitarios y múltiples radiales, disposición ligeramente en hileras radiales y tangenciales. Presencia de sustancias orgánicas en algunos vasos. Líneas vasculares en la sección tangencial, visibles a simple vista. Parénquima axial, visible con lupa de 1Ox, del tipo paratraqueal y escaso. Radios en la sección transversal visible con lupa, con espaciamiento y longitud regular, en la sección tangencial no estratificada, en la radial levemente contrastada.

\section{Características Microscópicas:}

Poros/vasos: Difusos, circulares a ovalados, espesor de la pared de $6.12 \mu \mathrm{m}$. Diámetro tangencial promedio $297,20 \mu \mathrm{rn}$, variando de $216.471 \mu \mathrm{m}$; clasificados corno grandes. De $2-7$ poros $/ \mathrm{mm}^{2}$, clasificados de muy pocos a poco numerosos, con mayor ocurrencia los pocos $(73,33 \%)$. Solitarios $(72,310 \%)$, múltiples de dos $(12,31 \%)$, múltiples de tres $(13,840 \%$ ), raro múltiples de 4 - 12 (Fig. 1). Longitud promedio de los elementos vasculares $588,511 \mathrm{~m}$, variando de $350-1000 \mu \mathrm{m}$; clasificados en medianos $(93,670 \%)$ y largos, con apéndice central en ambos extremos; raro en un solo extremo. Platina de perforación simple, horizontal a inclinadas (Fig. 10). Puntuaciones intervasculares de 5 - 9 $\mu \mathrm{m}$, pequeñas y medianas, de forma redonda, alterna, abertura inclusa; alargadas horizontalmente en algunos campos (Fig. 10, Fig. 11).

Radios: En la sección tangencial, altura promedio $223,71 \mu \mathrm{m}$, variando de 150 - $347 \mu \mathrm{m}$; clasificados en extremadamente cortos, de $8-20$ células de altura con mayor ocurrencia de 10 - 20 (77,670 \%). Exclusivo uniseriados. De 4 - 11 radios/ mm.; clasificados como poco 
abundantes. Distribuidos irregularmente en la sección tangencial, homogéneos, en algunos campos existen radios fusionados (Fig. 3). Puntuaciones radiovasculares similares a los intervasculares, en forma simples y escaleriformes (Fig. II).

Parénquima axial: Escasos, del tipo paratraqueal, vasicéntrico, levemente aliforme; presencia de grupos de células en bandas (Fig. 1, Fig. 3). En las secciones longitudinales, de 6-18 series/célula, cuadradas; algunas alargadas verticalmente (Fig.5).

Fibras: Del tipo libriforme, longitud promedio $1486,78 \mu \mathrm{m}$, variando de 1335 a $1963 \mu \mathrm{m}$; clasificadas en medianas (700\%) y largas. Diámetro total promedio 24,97 $\mu \mathrm{m}$, variando de 20-33; clasificadas de angostas (56,670 \%) a medianas. Diámetro promedio del lumen 17,75 $\mu \mathrm{m}$, variando de $12.27 \mu \mathrm{m}$. Espesor de la pared celular delgada, en promedio 3,56 $\mu \mathrm{m}$, variando de $2.4 \mu \mathrm{m}$. Puntuación simple, presencia de células bifurcadas en el extremo de algunas células (Fig. 7, Fig. 8, Fig.9).

Inclusiones: Presencia de cuerpos orgánicos no identificados en las células de los vasos y radios (Fig. 12).

\section{Estructura anatómica de la rama}

\section{Características Generales:}

Madera de densidad básica media $(0,56 \mathrm{gr} / \mathrm{cm})$. En condición verde, albura de color crema, duramen pardo claro. Seco al aire, la coloración se mantiene con pequeñas variaciones en la zona de transición entre albura y duramen. Olor y sabor no diferenciado. Brillo medio. Veteado no diferenciado. Grano recto. Textura media. Anillos de crecimiento poco diferenciado con bandas irregulares.

\section{Características Macroscópicas:}

Poros visibles a simple vista, difusos, solitarios en su mayoría con tendencia a disponerse en hileras tangenciales. Líneas vasculares visibles a simple vista. Parénquima axial ligeramente visible con lupa, escasos del tipo paratraqueal.

Radios en la sección transversal visible con lupa, con espaciamiento y longitud regular, en la sección tangencial son desordenados, en la radial levemente contrastados.

\section{Características Microscópicas:}

Poros/vasos: Difusos, circulares a ovalados, espesor de la pared celular de $6-12 \mu \mathrm{m}$, en algunas células con engrosamiento secundario en anillos. Diámetro tangencial promedio 
$219,86 \mu \mathrm{m}$, variando de $157-275 \mu \mathrm{m}$; clasificados en grandes $(66,670 \%$ y medianos. De $3-9$ poros $/ \mathrm{mm}^{2}$; clasificados en pocos $(66,670 \%$ y poco numerosos. Solitarios $(62,60 \%)$, múltiples de tres (17,30 o), múltiples de dos (12\%), raro múltiples de 4 . 12 (Fig. 2). Longitud promedio de elementos vasculares $372 \mu \mathrm{rn}$, variando de 300 - $450 \mathrm{ím}$; clasificados en cortos $(53,33 \%)$ y medianos, con apéndice central en ambos extremos; raro en un solo extremo. Platina de perforación simple, horizontales a inclinados (Fig. 6). Puntuaciones intervasculares de $6.8 \mu \mathrm{m}$ de diámetro, clasificados en pequeños y medianos, redondos y poligonales, alternos, abertura inclusa; en algunos campos alargadas y coalescentes (Fig. 6, Fig. 10).

Radios: En la sección tangencial de altura promedio $214 \mu \mathrm{m}$, variando de $142-331 \mu \mathrm{m}$; clasificados en extremadamente cortos. De 8 . 18 células de altura, más frecuentes de 10-20 $(60 \%)$. Uniseriados $(93,33 \%)$ biseriados $(6,33 \%)$. De 6- 10 radios $/ \mathrm{mm}$.; clasificados en poco abundantes. Distribuidos regularmente en la sección tangencial. Homogéneos, algunos heterogéneos, presencia de radios fusionados (Fig. 4, Fig. 6). Puntuación radiovascular similar a la intervascular (Fig. 11).

Parénquima axial: Escasos, del tipo paratraqueal vasicéntrico, levemente aliforme, presencia de grupos de células en bandas (Fig. 2). En las secciones longitudinales de 6.18 series por célula, cuadradas; algunas alargadas verticalmente. 
Figura 1. Corte transversal en el xilema del tronco

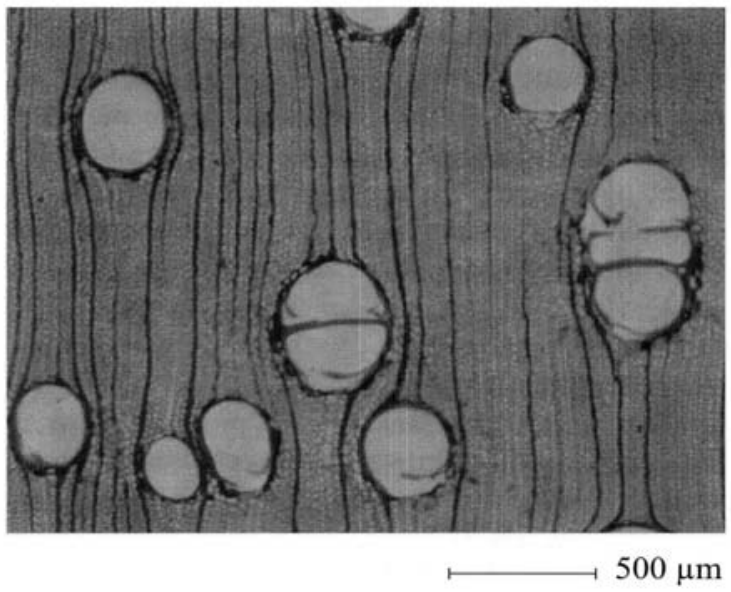

Figura 2. Corte transversal en el xilema de la rama

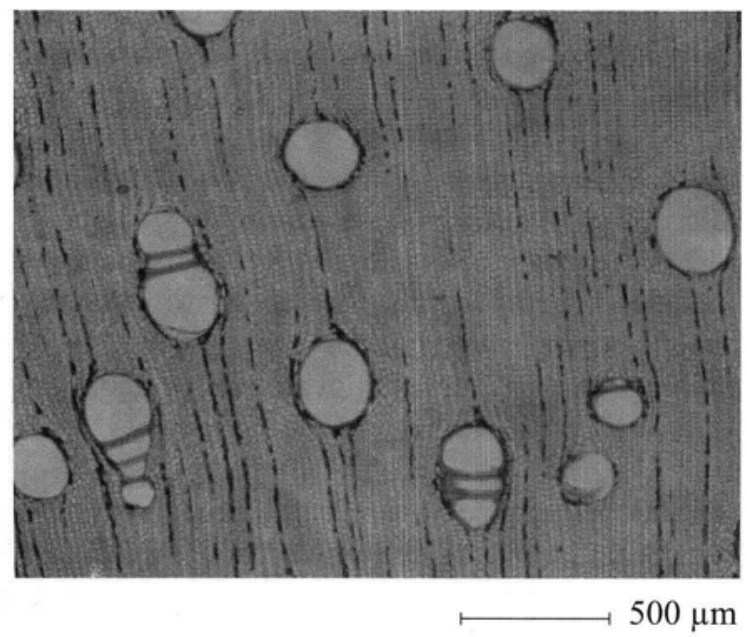


Figura 3. Corte radial en el xilema del tronco

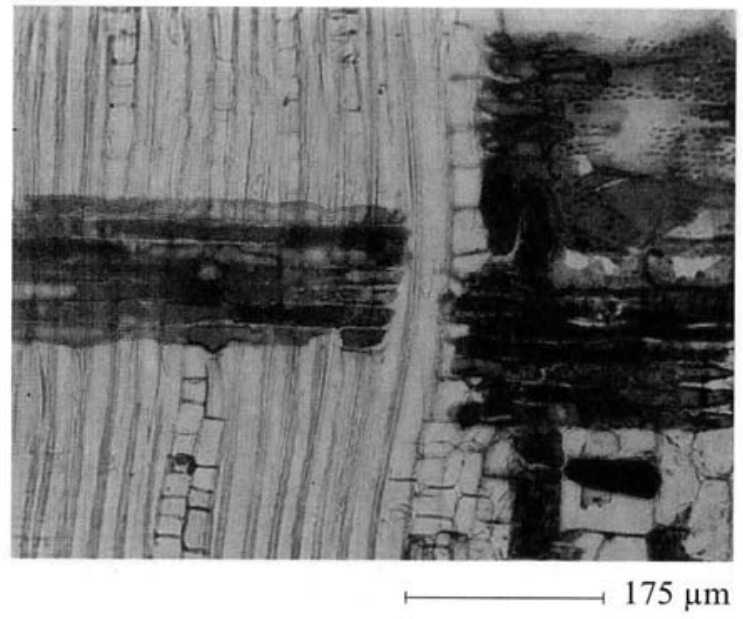

Figura 4. corte radial en el xilema de la rama

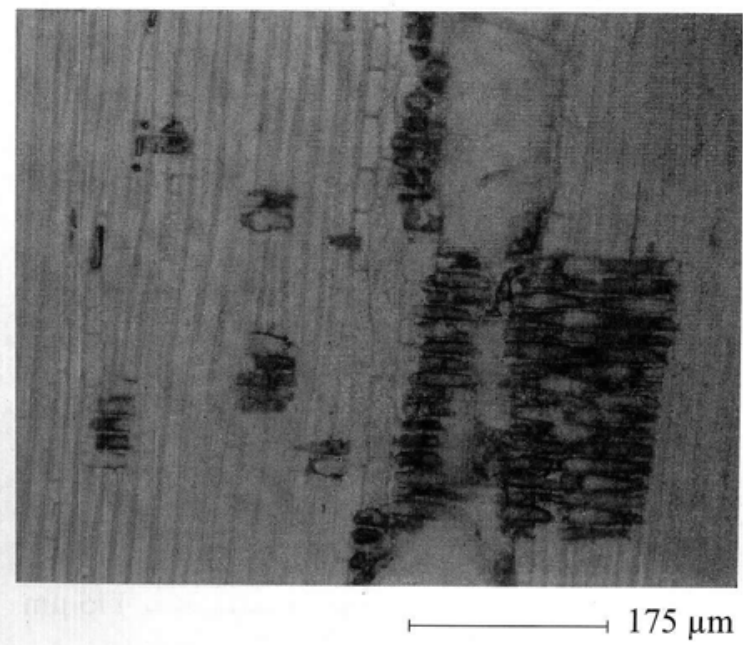


Figura 5. Corte tangencial en el xilema del tronco

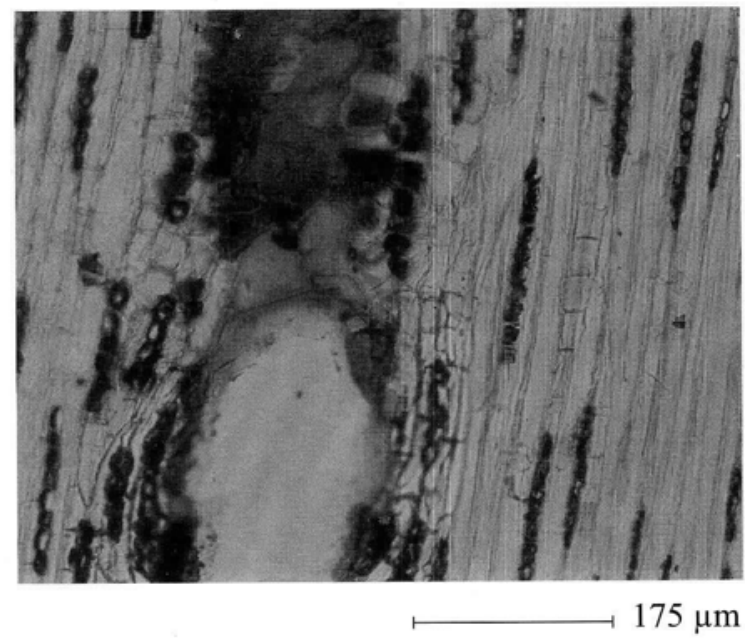

Figura 6. Corte tangencial en el xilema de la rama

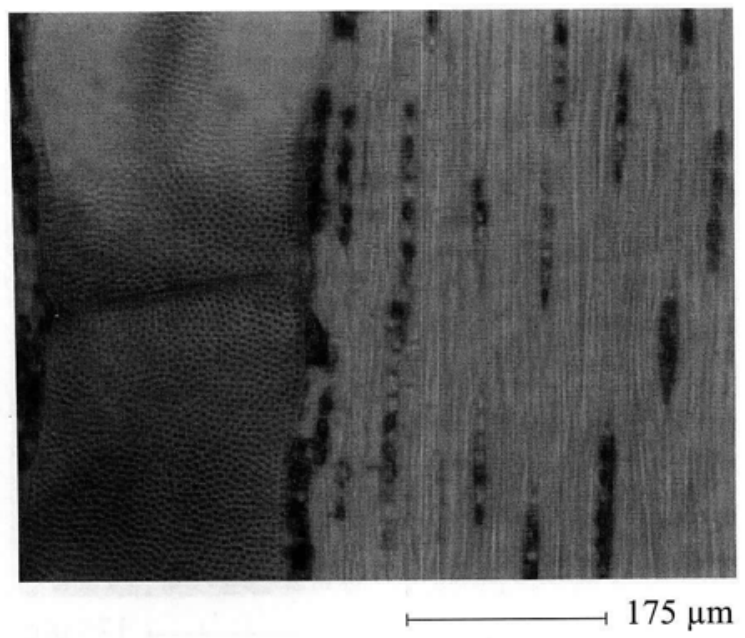


Figura 7. Fibra libriforme encontrada en el xilema del tronco y de la rama

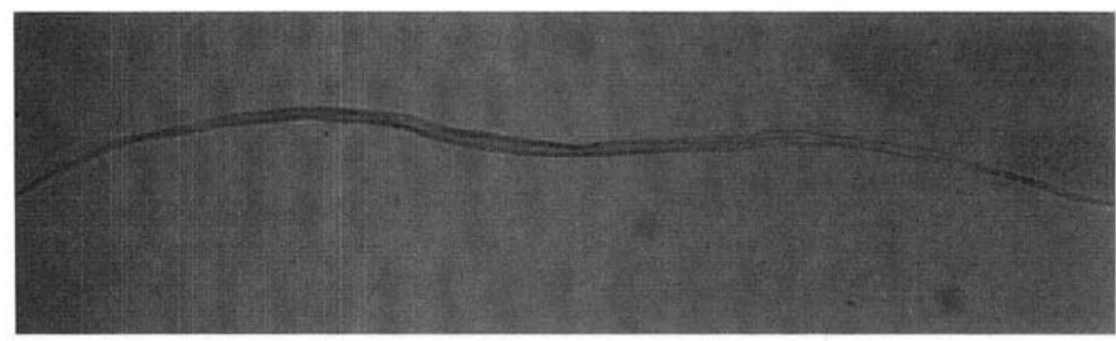

$175 \mu \mathrm{m}$

Figura 8. Fibra del tronco y de la rama con los extremos bifurcados

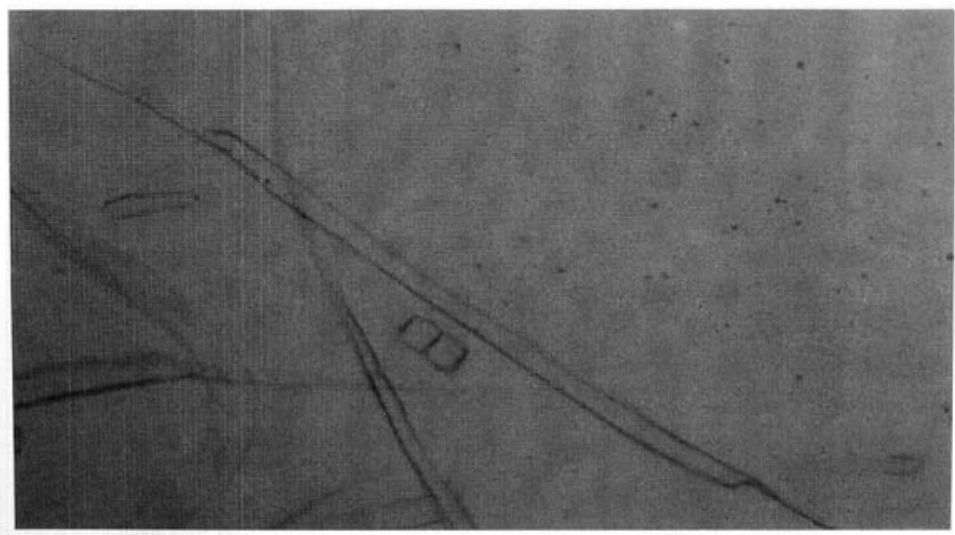


Fibras: libriforrnes, ligeramente estratificadas, longitud promedio $1281,05 \mathrm{im}$, variando de 1060- $1763 \mu \mathrm{m}$; clasificadas en medianas (90\%) y largas. Diámetro total promedio $18,78 \mu \mathrm{m}$, con los extremos de $10.25 \mu \mathrm{m}$; clasificadas en angostas $(86,67 \%)$ y medianas. Diámetro del lumen $11,77 \mu \mathrm{m}$, con los extremos de $3-20$ $\mu \mathrm{m}$. Pared celular delgada, en promedio 3,50 $\mu \mathrm{m}$, variando de 2-5 $\mu \mathrm{m}$ (Fig 7, Fi8). Puntuación simple, presencia de bifurcaciones en los extremos de algunas células (Fig. 8).

Inclusiones: Presencia de algunas sustancias orgánicas no identificadas en las células de los vasos y radios (Fig. 11).

Figura 9. (a) Punteaduras areoladas, (b) platina de perforación en los vasos

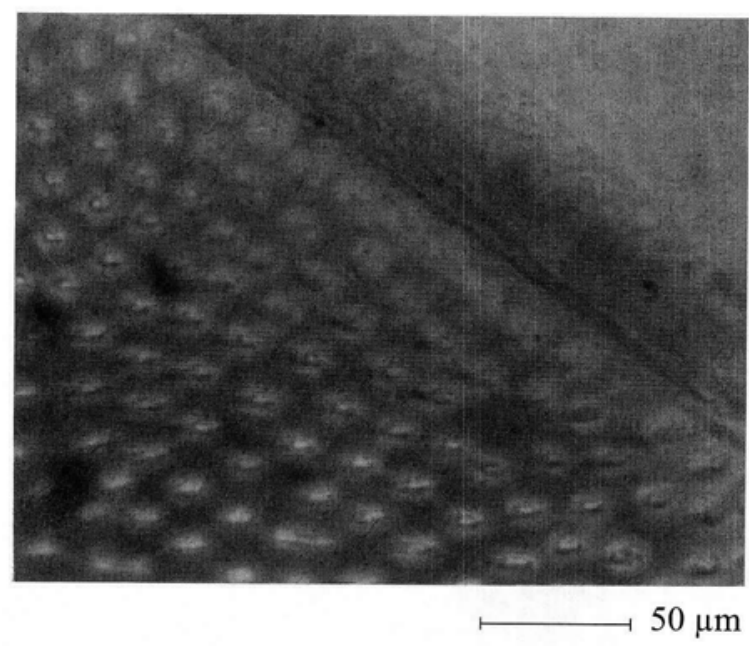


Figura 10. Campo de puntuación radiovascular en el xilema del tronco y rama

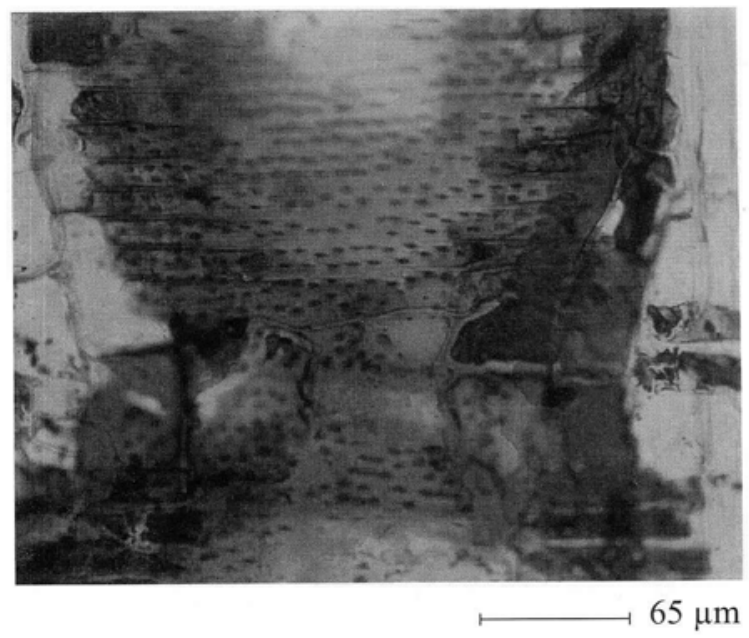

Figura 11. Compuesto orgánicos en los elementos vasculares del xilema del tronco y de la rama.

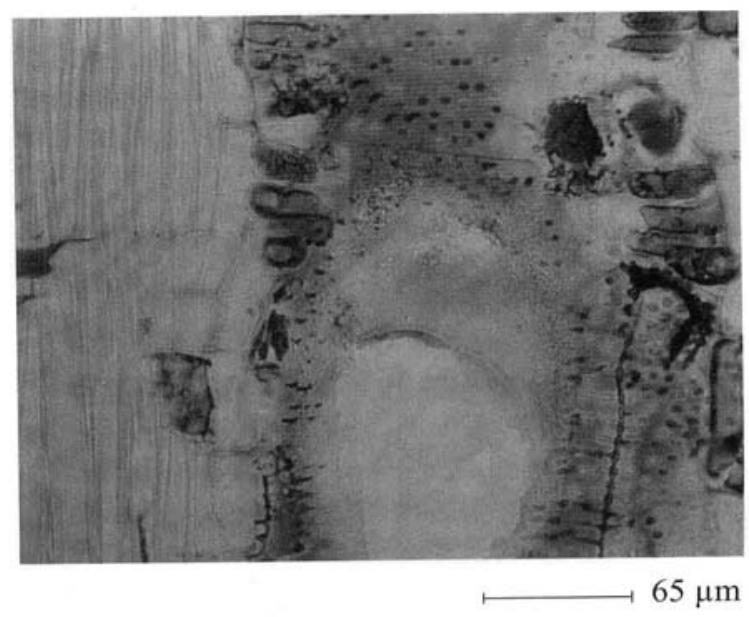


Figura 12. Engrosamientos secundarios de la pared celular en anillos en el xilema de las ramas

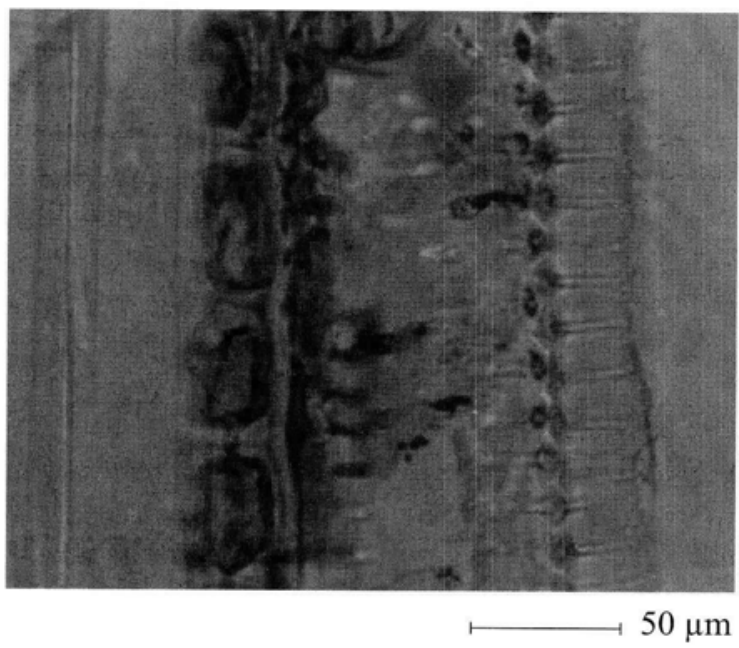

3. Características anatómicas diferenciales de la pared celular en anillos en el xilema de las ramas.

Las características anatómicas que diferencian la madera del tronco y de la rama se consigan en el cuadro $\mathrm{N}^{\circ} 1$ 


\section{Cuadro1. Características diferenciantes de ambas partes del árbol}

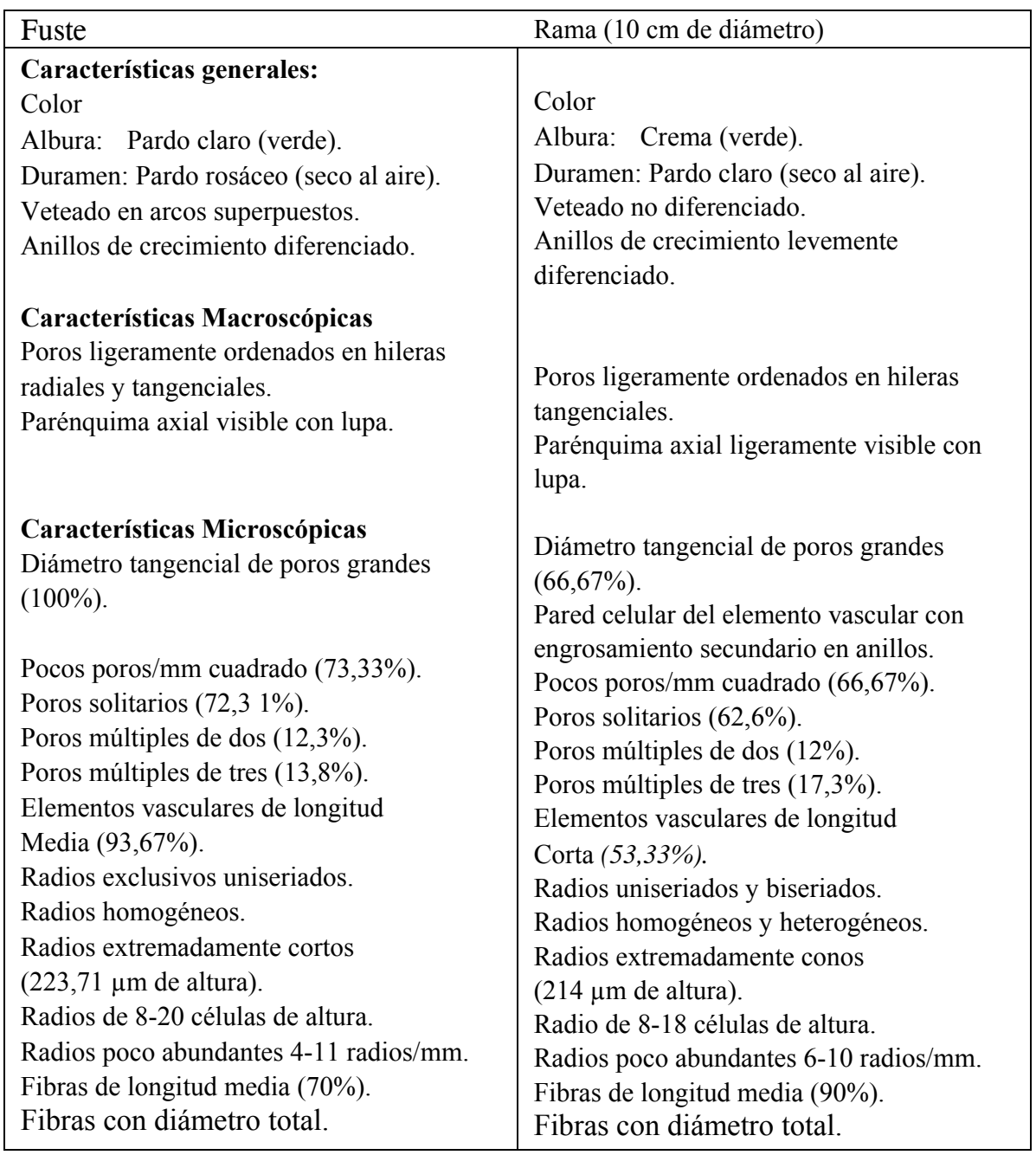

Existen características importantes que diferencian la madera de ambas partes del árbol; por ejemplo, la rama posee pared celular de vasos con engrosamiento secundario en anillos, lo cual no se observó en el xilema del tronco. 
Probablemente esta característica está influenciada por grupos de células en proceso de crecimiento existentes en la rama de $10 \mathrm{~cm}$ de diámetro, donde la superposición de partículas en forma de láminas está originando el engrosamiento de la pared celular del elemento vascular.

\section{Valores promedios de las mediciones}

Los Cuadros 2 y 3 presentan los valores promedio, coeficiente de variación y desviación estándar de las características cuantitativas de la madera de ambas partes del árbol.

\section{Cuadro 2. Características cuantitativas del tronco}

\begin{tabular}{|l|r|r|r|}
\hline Características & \multicolumn{1}{|c|}{$\mathrm{X}$} & $\mathrm{cv}(\%)$ & \multicolumn{1}{c|}{$\mathrm{s}$} \\
\hline & & & \\
Diámetro tangencial de poros $(\mu \mathrm{m})$ & 297,20 & 19,95 & 59,28 \\
Longitud de elementos vasculares $(\mu \mathrm{m})$ & 588,50 & 26,76 & 157,50 \\
Frecuencia de poros/mm cuadrado & 4 & 39 & 1,0 \\
Altura de radios $\left(\mathrm{n}^{\circ}\right.$ de células) & 14 & 23,3 & 3,0 \\
Altura de radios $(\mu \mathrm{m})$ & 223,71 & 21,73 & 50,78 \\
Ancho de radios $\mu \mathrm{m})$ & 16,56 & 10,45 & 1,73 \\
Frecuencia de radios/mm & 7 & 27,4 & 2 \\
Diámetro total de fibras $(\mu \mathrm{m})$ & 24,97 & 14,80 & 3,70 \\
Diámetro del lumen $(\mu \mathrm{m})$ & 17,75 & 22,54 & 4,0 \\
Espesor de pared celular de fibra $(\mu \mathrm{m})$ & 3,56 & 23,33 & 0,86 \\
Longitud de fibra & 1486,78 & 9.19 & 13662 \\
& & & \\
& & & \\
\hline
\end{tabular}

$\mathrm{x}=$ valor promedio, $\mathrm{s}=$ desviación estándar, $\mathrm{cv}=$ coeficiente de variación 


\section{Cuadro 3. Caracterización cuantitativa de la rama}

\begin{tabular}{|l|r|r|r|}
\hline Características & x & cv (\%) & \multicolumn{1}{c|}{ S } \\
\hline & & & \\
Diámetro tangencial de poros $(\mu \mathrm{m})$ & 219,86 & 15,18 & 33,3 \\
Longitud de elementos vasculares $(\mu \mathrm{rn})$ & 372,00 & 12,10 & 45,0 \\
Frecuencia de poros/mm cuadrado & 5 & 40,51 & 2,0 \\
Altura de radios $\left(\mathrm{n}^{\circ}\right.$ de células) & 11 & 30,39 & 3,4 \\
Altura de radios $(\mu \mathrm{m})$ & 214,00 & 21,37 & 45,7 \\
Ancho de radios $(\mu \mathrm{m})$ & 17,35 & 14,52 & 2,5 \\
Frecuencia de radios/mm & 8 & 14,53 & $\mathbf{1 , 1}$ \\
Diámetro total de fibras $(\mu \mathrm{m})$ & 18,78 & 24,47 & 4,6 \\
Diámetro del lumen $(\mu \mathrm{m})$ & 11,77 & 41,70 & 4,9 \\
Espesor de pared celular de fibra $(\mu \mathrm{m})$ & 3,50 & 25,71 & $\mathbf{0 , 9}$ \\
Longitud de fibra & 1281,05 & 16,40 & 210 \\
& & & \\
& & & \\
\hline
\end{tabular}

$\mathrm{x}=$ valor promedio, $\mathrm{s}=$ desviación estándar $\mathrm{cv}=$ coeficiente de variación

Se observa que a excepción de la pared celular de fibras, la comparación de medias al nivel de 500 de probabilidad determina que los valores promedios difieren significativamente, lo que indica diferencias entre las características cuantitativas del tronco y de la rama de 10 $\mathrm{cm}$. Esta diferencia estadística no afecta la clasificación de la madera por el tamaño de las células; por ejemplo, ambas partes del árbol fueron clasificadas como madera de poros grandes, a pesar que la presencia de poros grandes en el tronco es más frecuente que en las ramas; de igual manera posee fibras de longitud mediana, con mayor frecuencia en las ramas $(90 \%)$.

Los resultados indican que el diámetro tangencial de los poros y la longitud de los elementos vasculares en el tronco son 26 y $36,7 \%$, respectivamente, más grandes que en las ramas. Los radios de la madera del tronco observados en la sección tangencial son 3400 más altos que lo observado en las ramas. Las fibras en todas sus dimensiones son mayores en el tronco que en la rama: 28,700 para el diámetro total, 33,8\% para el diámetro del lumen, 1,680 o para el espesor de la pared celular y $13,80 \%$ para su longitud. 


\section{Aptitud de la madera para la clasificación de pulpa para el papel}

\begin{tabular}{|l|l|l|}
\hline Parte del árbol & Factor R & Clasificación \\
\hline Tronco & 0,40 & Muy buena para el papel \\
Rama & 0,56 & Buena para papel \\
& & \\
\hline
\end{tabular}

Estos resultados indican que ambas partes del árbol probablemente puedan ser utilizadas para fabricar pulpa para papel. A pesar de las diferencias en la estructura celular por el tamaño de fibras, el factor Runkel toma en consideración el espesor de la pared celular y diámetro del lumen independientemente de otros factores; sin embargo, la calidad de pulpeo puede mejorar utilizando el método adecuado en el proceso de fabricación.

\section{CONCLUSIONES}

Existen características cualitativas y cuantitativas comunes en ambas partes del árbol de la especie Cedrelinga catenaeformis D., que pueden servir como elemento de identificación y clasificación anatómica de la madera, basado en la rama de $10 \mathrm{~cm}$ de diámetro.

Las características cualitativas y cuantitativas que diferencian a ambas partes del árbol, se encuentran consignadas en los Cuadros 1,2 y 3.

Al nivel de 5\% de significancia, las características cuantitativas de ambas partes del árbol son, estadísticamente, diferentes.

La clasificación anatómica de la madera por sus características cuantitativas fue similar en ambas partes del árbol.

Por el tamaño de fibras, ambas partes del árbol fueron calificadas como aptas para ser utilizadas en pulpa para papel.

\section{AGRADECIMIENTO}

A los profesionales Angel Angulo Ruiz, Oscar del Aguila Rengifo y Jarlin Arellano Valderrama, por el apoyo decidido en la ejecución del presente trabajo. 


\section{BIBLIOGRAFIA}

ALENCAR, J. C. y ARAUJO, V. C. de. 1971. Comportamento de espécies florestais quanto a luminosidade. En: Acta Amazonica, V. 10, No 3, pp. 435-444.

AROSTEGUI, A. y DIAZ, M. 1992. Propagación de especies Jbrestaies nativas promisorias en Jenaro Herrera. iquitos: IIAP/CIJH, 119 pp.

BHAT, K. M. y DHAMODARAN, E. K. 1989. Fibre length variation in stem and branches of eleven tropical hardwoods. En: JAWA Bulletin ns. V. 10, No 1, pp.63-70.

CAMACHO, P. y CANESSA, E. 1981. Determinación de las características macroscópicas y microscópicas, padrones de variación dc fibras de especies tropicales y su efecto en la elaboración de pulpa para papel. Instituto Tecnológico de Costa Rica. 152 pp.

COMISION PANAMERICANA DE NORMAS TECNICAS (Copant). 1972. Maderas.

Selección y colección de muestras. Norma Copant, $\mathrm{N}^{\circ}$ 458, pp. 1-11.

-1973. Maderas. Descripción de características organolépticas, macroscópicas, y microscópicas de dicotiledóneas angiospermas. Norma Copant, $\mathrm{N}^{\mathrm{o}} 30$, pp.1 09.

DENNE, M. P. y DODD, R. 5. 1989. Control of variation in wood quality within hardwood and softwood trees. M. TT Bunder fors Chunggsanstalt f. Forts-M. Holzwirtschaft. $\mathrm{N}^{\mathrm{o}} 131$, pp. $7-31$.

FREITAS, M.; MEDEIROS, L. y LIMA, A. de. 1992. Leguminosas da Amazónia Brasileira II. Cedrelinga Ducke (Leg . Mimos). En: Bol. Mus Para. Emilio Goeldi. Ser Bot., V. 8, Nº1, pp. 143-156.

IAWA, Committee on Nomenclature. 1964. Multilingual glossaly oí terins used in wood anatonw. Konkordias, Winterthur, Switzerland.

IAWA Committee. 1989. List of Microscopic features for hardwood identification. En: IAWA Bulletin ns. V. 10, No 3, pp. 2 19-332. 
IQBAL, M. y GHOUSE, A. K. M. 1983. Analytical study on cell size variations in sotne arid trees of India: Acacia nilotica and Prosopis spicigera. En: IAWA Bulletin ns. V. 4, $\mathrm{N}^{\mathrm{o}} 1$, pp. ${ }^{46-52 .}$

KLUNGNESS, J. H. y SANYER, N. 1981. Hardwood pulp utilization: separation of rnonifibrous oak cornponents. En: Tappi 64(3), pp.109-113

KORT, 1. de. 1990. Tracheid length in vital and non vital douglas Fir (Pseudotsuga menziesii) in the Netherlands. En: JAWA Bulletin ns. V. 11, No 2, pp. 203-209.

LEE, P. W. y EOM, Y. G. 1988. Anatomical comparisombetween compressionwood and opposite wood in a branch of korean pine (Pinus koraiensis). En: IAWA Bulletin ns. V. 9, No 3, pp. 275-284.

LINARES, C. 1986. Rada les y huertos semilleros en bosques tropicales. Tarapoto (Perú): IIAP, $35 \mathrm{pp}$.

LLUNCOR, D. 1977. Relaciones entre las características de cepillado en algunas maderas de Venezuela y sus propiedades físico mecánicas y anatómicas. Tesis de post-grado. Venezuela: Universidad de los Andes. Facultad de Ciencias Forestales. $90 \mathrm{pp}$.

MAEGLIN, R. R. y QUIRK, J. T. 1984. Tissue proportions and cell dimensions for red and white oak groups. En: Can J. For Nes, V. 14, pp. 101-106.

MAGALHAES, L. M. 5. y BLUM, W. H. 1984. Nodulaçậo e crescimento de Cedrelinga catenacformis Ducke em plantíos experimentais na regiáo de Manaus. En: Pesq. Agron. Bras. V. 19, pp. 159-164.

MANWILLER, F. 1974. Fiber lengths in stems and branches of small hardwoods on southern pine sites. En: WoodScience. V. 7, No 2, pp. 130-132.

OFICINA NACIONAL DE EVALUACION DE RECURSOS NATURALES. (ONERN) 1975. Inventario, evaluación e integración de los Recursos Naturales de la zona de Iquitos, Nauta, Requena y Colonia Anganios. Lima: 334 pp. 1976. Mapa Ecológico del Perú. Guía explicativa. Lima. 147 pp. 
PAGE, V. M. 1993. Anatomical variation in the wood of Robinia pseudoacacia L. and the identify of miocene fossil woods from Sourthwcstern LJnited States. En: IAWA Builetin os. V. 14, No 3, pp. 299-3 14.

PARK, S.J.; SAIKI, H. y HARADA, H. 1979. Stnicture of branch wood in Akamatsu (Pinos densijlora 5. et. 7)1. Distribution of compression wood, structure of annual ring and tracheids. En: Mokuzai Gakkaishi. V. 25, pp. 311- 317.

1980. Ibid II Well structute of branch wood tracheids. Mon Coll. En: Agi: Kyoto Univ., V. 115, pp. 33-44.

PAULA, J. E. 1980. Estudo anatómico das madeiras de Vro/a sebiféra Aubl. e Pseudobonihax tomentosuni (Mart et Zucc). a. Robyna visado o seu aproveita mento tecnológico. En: Brasil Floresta!, No 42, pp. 35-52.

SPICHIGER, R.; MEROZ, J.; LOIZEAU, P. y STUTZ DE ORTEGA, L. 1989. Contribución u la flora de la A,nazon ¿a Peruana; Los árboles del arboretum de Jenaro Herrera. Conservatorio y Jardín Botánico de Ginebra/COTESU/IIAP, V. 1.359 pp.

STOKE, D. D. y MANWILLER, E. G. 1994. Proportions of wood elements in stern, brandi, and root wood of black oak (Quercus velittina). En: JAWA Bulletin 12.5V. 15, N 3 , pp. 301-310.

TOSI, J. A. 1960. Zonas de vida natural del Perú. Lima: IIGA-OEA, 271 pp.

TRUJILLO, T. y GONZALES, R. 1985. Durabilidad natural de ocho especies forestales del Perú, medio nutritivo natural. En: Revista Forestal del Perú. V. 13, No 1, pp. 63-69.

VALDERRAMA, H.; LOUREIRO, A. y AROSTEGUI, A. 1989. Estructura anatamica y clave de ideo tjicación de veinte especies fórestales de la zona de Colonia Angamos, río Yavaríy Jenaro Herrera. Iquitos: Convenio INPA/IJNAP/ IIAP. 130 pp.

VALDERRAMA, H. 1992. influencia de la estructura anatómica en el comportamiento tecnológico de treinta especies forestales de la Amazonia Peruana. En:

Conocimiento. V. 2, $\mathrm{N}^{\mathrm{o}} 2-3$, pp. 13-23. 
WRIGHT, J. A. y MALAN, F. S. 1991. Variation in wood and tracheid properties of Pinus maxírninoi, Pinus pseudostrobus and Pinus patuIa . En: JAWA Bulletin n.s. V. $12, \mathrm{~N}^{\circ} 4$, pp. $467-475$.

YAMAMOTO, A. K. 1974. Secagern da madeira. Instituto de Pesquisas

Tecnológicas do Estado de Sáo Paulo. En: Boletin Técnico, V. 2, No 1, pp. 85-145. 